\title{
Clinical Assessment of Inlay-retained Bridge Designs (Tub-shaped and Inlay-shaped) in Missing Posterior Teeth Cases: A Randomized Controlled Trial
}

\author{
Mohamed MA Abdelfattah ${ }^{1}$, Omaima S El Dein El Mahallawi ${ }^{2}$, Ahmed N Abdelaziz ${ }^{3}$
}

\begin{abstract}
Aim: This study was aimed to clinically assess the two inlay retained bridges (tub- and inlay-shaped designs) in missing posterior teeth cases. Materials and methods: Thirty cases with missing single posterior tooth were included in the trial and divided into two main groups with a 1:1 allocation ratio ( $n=15$ for each group). Group I (the control group) received an inlay-retained bridge with inlay design on both abutments. Group II (the intervention group) received an inlay-retained bridge with tub design on both abutments. The inlay-retained bridges were fabricated from monolithic zirconia and bonded using dual-cured self-adhesive resin cement after treating the fitting surfaces mechanically and chemically. The clinical evaluation of the retention, marginal adaptation, and secondary caries occurrence was commenced following the Modified United States Public Health Service (MUSPHS) criteria 12 months after cementation.

Results: The results show that the difference was not statistically significant between both groups regarding retention, marginal adaptation, and secondary caries occurrence after 12 months of cementation, with 0.05 statistical significance level and confidence interval of $95 \%$.

Conclusion: Within the limitations of this trial, the retention, the marginal adaptation, and the secondary caries for both inlay-retained bridge designs exhibited comparable outcomes.

Clinical significance: The use of the inlay-retained bridges with tub design can be superior to the inlay-retained bridges with inlay design regarding the conservation of the tooth structure with the same retention rate, marginal adaptation, and occurrence of secondary caries in missed posterior tooth cases.
\end{abstract}

Keywords: Fixed partial denture, Marginal fit, Randomized controlled trial, Resin cements, Retention, Secondary caries and zirconia.

World Journal of Dentistry (2020): 10.5005/jp-journals-10015-1713

\section{INTRODUCTION}

Recent evolution of the adhesive restorative materials and techniques has provided the clinicians with plenty of conservative treatment options that preserve tooth structure and at the same time improving the longevity and esthetics of the prosthesis. The reduced invasiveness of these resin-bonded adhesive restorations makes them an appealing alternative to conventional restorations. ${ }^{1}$

Zirconia restorations used for dental purposes can be fabricated using CAD-CAM techniques by two methods. The first involves milling a zirconia block which is fully sintered with no shrinkage to the milled prosthesis. The drawbacks include the greater wear of the milling burs and the presence of flaws generated throughout the grinding procedure which compromise the mechanical qualities of the prosthesis. The second involves milling the restorations using a block which is pre-sintered, meeting the maximum strength once sintered, where the contraction of the prosthesis can be counteracted during designing the restoration, so that the prosthesis fit is guaranteed. The two methods encompass the following primary procedures: digital scanning, prosthesis designing using software and prosthesis milling. The zirconia restorations constructed through the CAD-CAM technology possess sufficient accuracy to be used in the dental field. ${ }^{2}$

Monolithic zirconia restorations were developed to overcome the drawbacks related to the veneered restorations, such as the high rate of fracture of the veneering porcelain $(6-25 \%$ after 3 years), which was reported for zirconia more than that for metal veneered restorations. These results promote few researchers to
${ }^{1}$ Fixed Prosthodontics Department, Faculty of Dentistry, Cairo University, Cairo, Egypt

${ }^{2,3}$ Department of Fixed Prosthodontics, Cairo University, Cairo, Egypt

Corresponding Author: Mohamed MA Abdelfattah, Fixed Prosthodontics Department, Faculty of Dentistry, Cairo University, Cairo, Egypt, Phone:+20 1221583708,e-mail:mohamed.abdelgawad@ dentistry.cu.edu.eg

How to cite this article: Abdelfattah MMA, El Dein El Mahallawi OS, Abdelaziz AN. Clinical Assessment of Inlay-retained Bridge Designs (Tub-shaped and Inlay-shaped) in Missing Posterior Teeth Cases: A Randomized Controlled Trial. World J Dent 2020;11(2):121-127.

Source of support: Nil

Conflict of interest: None

evaluate the fracture rate of all-ceramic restorations, indicating that delamination and chipping are the most common modes of failure of these types of restorations. ${ }^{3}$

The optimal surface treatment for bonding to zirconia is either chemical and/or mechanical, and these different types of treatments play an important role in constructing a successful zirconia restoration. While zirconia usage for dental prosthetics is not uncommon, the superior treatment that produces a strong bonding of zirconia to the prepared teeth surfaces yet remains unknown. Dentists are sometimes distracted with regard to the best method needed for zirconia to be treated with, prior to bonding. This happened because there were no clear illustrations in the 
literature, from dental manufacturers, on how to treat zirconia properly to produce a durable bond. ${ }^{4}$

As a result, this trial will benefit clinicians by guiding them to choose a more conservative treatment option with superior fit values which will return to the patient with prolonged survival of the restoration, less chair time, and less risk for pulp injury, indicating more successful restoration. Consequently, this study aimed to clinically assess two inlay bridge designs (tub and inlay shaped) in missed posterior teeth cases in a controlled randomized study. The clinical assessment of the retention, marginal adaptation, and secondary caries were done following the MUSPHS criteria after 12 months of cementation.

\section{Materials and Methods}

\section{Trial Design}

Controlled randomized parallel group clinical trial.

\section{Trial Population}

This trial was conducted on patients with missed single posterior tooth cases from the outpatient clinic in The Fixed Prosthodontics Clinic, Faculty of Dentistry, Cairo University. This study was conducted between April 2018 and August 2019. All subjects were required to be aged between 18 years and 55 years, withstand the prosthetic process (physically and psychologically), sign the consent form, with good dental and periodontal health, sufficient crown height (minimally $5 \mathrm{~mm}$ ), proper alignment of the abutment teeth, and enough measurements of the area of the missed tooth. Exclusion criteria included partially erupted teeth, patient with bad oral hygiene, nonvital tooth, psychotic disturbance or unreal needs of the patients, abnormal occlusal activities, and existence of insufficient enamel at the margins of the prepared teeth. A total of 30 cases were selected and divided into two main groups with a 1:1 allocation ratio ( $n=15$ for each group) according to the inlay bridge design fabricated. The patients selected were randomly allocated into two groups using random sequence generator, the control group (inlay-shaped design group), and the intervention group (tub-shaped design group).

\section{Clinical Study}

Detailed preoperative data were collected from all patients including personal data, medical history, dental history, intraoral, extraoral, and radiographic examination. Before teeth preparation, full mouth scaling, shade selection, primary impressions with alginate impression material (Hydrogum, Zhermach, Italy) and photographs were taken.

Teeth preparations were done as follows:

\section{Control Group}

- The inlay-shaped design involved occlusal and proximal box preparation with round line angles, corners and a rectangular floor. The occlusal preparation permits a $2.5 \mathrm{~mm}$ depth and $4 \mathrm{~mm}$ width of zirconia, with approximately $6^{\circ}$ divergence of the walls. The proximal box was prepared with $1 \mathrm{~mm}$ width and $2 \mathrm{~mm}$ extension apical to the floor of the isthmus with approximately $6^{\circ}$ divergence. This will contribute to a $4.5 \times 4 \mathrm{~mm}$ connector dimension. In case of caries that are extended beyond the preparation margin, the case was excluded and referred to the conservative department for restoration.

\section{Intervention Group}

- The tub-shaped design involved occlusal surface preparations as the inlay design but without the preparation of the proximal box, with $2.5 \mathrm{~mm}$ occlusal cavity preparation depth.

All preparations were done using a blue-coded high-speed tapered diamond stone with flat end (Brasseler, USA) by the same operator for both designs (Fig. 1).

The preparations were finished using yellow-coded tapered diamond stone with flat end finishing stones. Using a stock tray, a two-step double-mix final impression technique was made using addition silicon (Elite HD+; Zhermack, Italy). Bite records were done using polyvinyl siloxane bite registration material (Imprint 4 bite registration VPS; $3 \mathrm{M}$ ESPE, USA), and temporary filling (Coltene, Spain) was placed in the cavity. A master cast was constructed after pouring the impression with a type IV dental stone, according to the manufacturer's instructions.

\section{Bridge Fabrication}

\section{Scanning the Casts}

Identico T300 Scanner (DentCore, USA) was used for both arches separately and in occlusion. Merge for each individual scanned cast was done with the corresponding cast in the occlusion scan.

\section{Designing the Restoration}

After scanning, the captured pictures were preserved in the program archive. The program computed a digital cast after scanning using the saved images. The preparation edges were detected manually, the bridge insertion axis was detected automatically by the program and the missed tooth was placed manually.

\section{Milling Process}

To start the milling procedure, the type of the block (Katana multilayer zirconia disc, Noritake, Japan), as well as the scale factor (1.25) of the disc, was entered manually and verified by selecting the $(\sqrt{ })$ mark. Properly sized zirconia disc was selected and placed in the milling chamber of the Roland DWX-510 milling machine (Roland DGA Corporation, USA) fastened with the set screw. Then the restorations were detached from the block using an abrasive

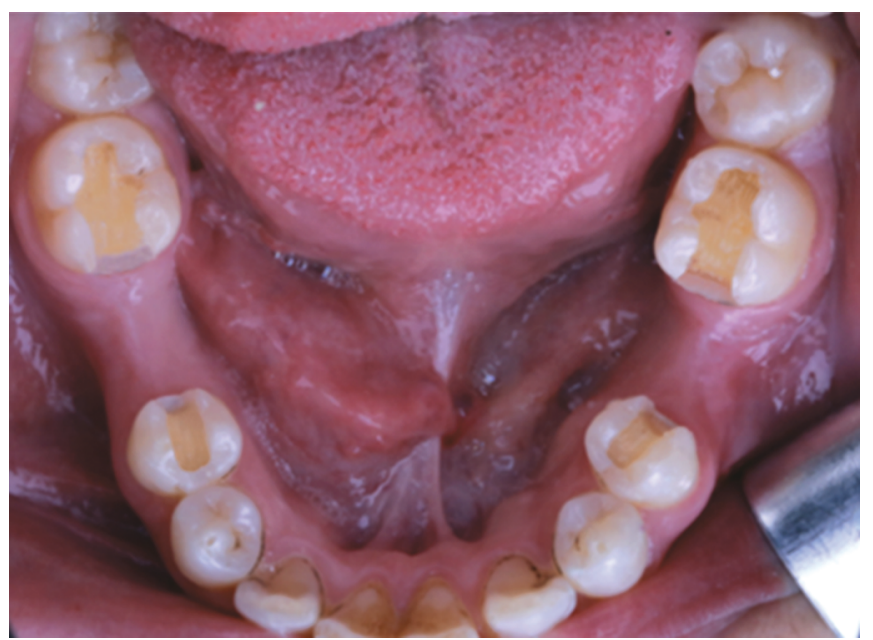

Fig. 1:Tub-shaped (right side) and inlay-shaped (left side) preparation design 
cutting diamond tool. After finalizing the milling procedure, the bridges were placed in an ultrasonic cleaner for 1 minute and then completely dried before sintering (Fig. 2).

\section{Sintering of the Bridges}

Sintering was conducted using the Mihm Vogt Tabeo Sintering furnace (Panadent, UK). The pre-sintered zirconia bridges were placed on to the crucible rack after filling it with a layer of sintering beads with the occlusal surface facing down. The sintering tray was placed on the shelf in the furnace. The desired program was selected according to the manufacturer instructions, then the furnace door closed manually, and the program was activated by pushing the start button. After the cycle was completed, the furnace door was opened, the sintering tray was lifted and the bench cooled on the crucible rack. Inspection of the bridges for any flaws, defects, and cracks was done.

\section{Checking and Verification of the Restorations}

The temporary restorations were removed using excavator; cotton rolls were used to isolate the prepared teeth. Abutment teeth were cleansed with pumice.

\section{Seating}

The bridges were seated with reasonable finger pressure to ensure proper seating.

\section{Marginal Integrity}

It was determined by moving a sharp explorer from the tooth to the restoration and from the restoration to the tooth. If resistance was encountered in any direction, this would be an indication for a gap or open margin indicating remake of the bridge.

\section{Retention}

Retention is the resistance to vertical displacement. The restoration was evaluated to be self-retentive and not depending on the cement.

\section{Stability}

Stability is the resistance to lateral displacement (rocking and rotation). It was checked by placing the framework in place and by one finger on one terminal retainer to detect any rocking or rotation in the other retainer.

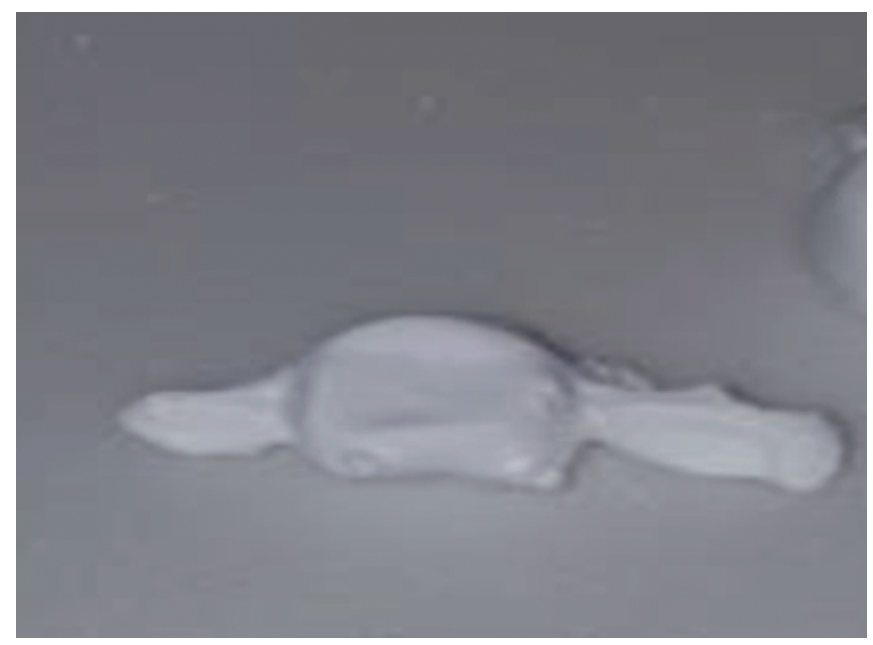

Fig. 2: Pre-sintered zirconia bridge

\section{Occlusion}

Occlusion was checked using Shim stock of $8 \mu \mathrm{m}$ thickness, which was grasped and put between the restoration and the opposing teeth and pulled while the teeth in occlusion, to ensure that the restoration is not out of occlusion. Then the Shim stock was grasped as before, but this time, it was placed on the opposite side and pulled while the teeth in occlusion to ensure that the restoration has no high spots. Articulating paper with $35 \mu \mathrm{m}$ thickness was then used to detect any centric or eccentric premature contacts which were removed using diamond stone and polished using zirconia polishing kit.

Pontic

Superfloss was used to ensure proper pontic-ridge relation.

\section{Connectors}

Connectors were evaluated to ensure that they were not blocking the gingival or occlusal embrasures.

\section{Staining of the Bridges}

Staining was conducted using the Vita Akzent plus staining kit (VITA Zahnfabrik, Germany). This was done using the Programat P510 ceramic furnace (Ivoclar Vivadent, Germany) at $850^{\circ} \mathrm{C}$ firing cycle.

\section{Surface Treatment of Zirconia}

Two types of surface treatment of zirconia were conducted:

\section{Mechanical Surface Treatment}

- Cleaning the zirconia surfaces from saliva remnants.After try-in, the restorations were rinsed with water spray and dried with air. All bonded surfaces of the restoration were covered with a layer of ZirClean (Bisco, USA) and left for 20 seconds, then the restorations were rinsed thoroughly with water spray and dried with air.

- The fitting surfaces of the inlay bridges were sandblasted with $\mathrm{Al}_{2} \mathrm{O}_{3}$ particles with $50 \mu \mathrm{m}$ diameter, $2.8 \mathrm{bar}$, and $1 \mathrm{~cm}$ distance, water sprayed for 60 seconds and cleaned using the ultrasonic cleaner in $95 \%$ ethyl alcohol for 10 minutes.

\section{Chemical Surface Treatment}

On the fitting surfaces of zirconia bridges, 10-methacryloyloxydecyl dihydrogen phosphate containing primer (Z Prime Plus, Bisco, USA) was applied.

\section{Surface Treatment of the Abutment Teeth}

No special surface treatment was needed for the abutment teeth as the type of cement used was self-adhesive resin cement.

\section{Final Cementation of the Restoration}

The dual-cure self-adhesive resin cement (TheraCem; Bisco, USA) was applied to the intaglio surfaces of the bridges and to the preparation surfaces. The restorations were first placed in the site with finger pressure. Excess cement was carefully removed using a brush. A layer of glycerin gel was placed to avoid the formation of an air inhibited layer; this was followed by light curing at the four axial line angles and in occlusal direction for 1 minute. Finishing and polishing of the margins were commenced using finishing diamond burs, rubber polishing points, and diamond polishing paste (Fig. 3). 


\section{Follow-up Procedure}

Evaluation of the restorations' retention, marginal adaptation, and recurrent caries were done after 12 months by an independent assessor following the MUSPHS criteria.

\section{Retention}

Retention was evaluated using sharp dental explorer. The retention score was "Alpha" if the restoration is present in place or "Charlie" if the restoration is partially or totally debonded.

\section{Marginal Adaptation}

Marginal adaptation was evaluated by passing sharp dental explorer across the surface of the restoration toward the tooth. The score was Alpha if there was no catch by the explorer, "Bravo" if there was a catch or charlie if the explorer penetrates into crevice defect extended to the dentoenamel junction.

\section{Recurrent Caries}

Recurrent caries was evaluated by inspecting the margin area for discoloration. Alpha score if the bridge is continuous with the normal tooth anatomy. In case of a dark deep discoloration visible alongside the bridge, a Charlie score is given.

\section{Statistical Analysis}

The retention rate throughout the control group was $52 \%$ as reported by OhImann et al. ${ }^{5}$ and Deniz et al. ${ }^{6}$ Provided that the

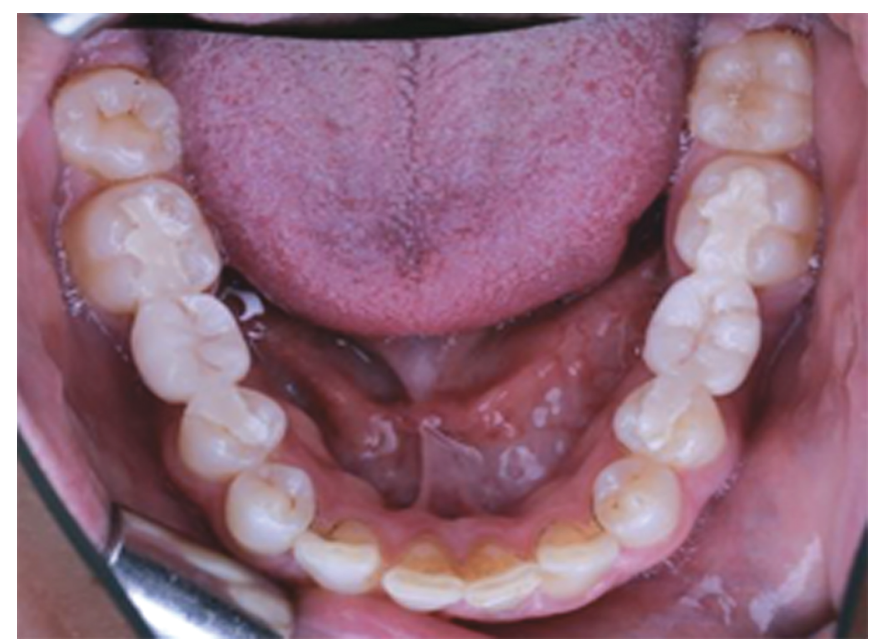

Fig. 3: Final cementation true retention rate is $99 \%$ among the intervention group, and to be able to refuse the null hypothesis (the debonding rate is equal to the probability (power) 0.8 for both study groups), 12 inlay-retained bridges needed to be studied in each group. The type I probability error correlated with the test of this null hypothesis is 0.05 . The null hypothesis will be assessed using uncorrected chi-square statistics. To counteract the possibility of losses of participants throughout the follow-up period, the number of participants can be increased by $25 \%$ of the calculated number, so that the number will be 15 . The software used for calculation of the sample size was the PS: Power and Sample Size Calculation software, Version 3.1.2 (Vanderbilt University, Nashville, Tennessee, USA).

\section{Results}

Frequency (count) and relative frequency (percentage) were used to summarize the categorical data. A Chi-square $\left(\chi^{2}\right)$ test was used to compare the categorical data. Exact test was used if the expected frequency is less than 5 . Statistically significant difference was considered if the $p$ value $<0.05$.

Descriptive statistical analysis presented in Table 1 and Fig. 4 revealed the following according to the MUSPHS criteria after 1 year of evaluation:

- Regarding retention, the inlay retained bridges with inlay design exhibited a $93.3 \%$ retention rate, while for the tub design, the retention rate was $80 \%$, and this difference was not statistically significant.

- Regarding marginal adaptation, the inlay retained bridges with inlay design and the inlay retained bridges with tub design exhibited $100 \%$ marginal adaptation.

- Regarding secondary caries, the inlay retained bridges with inlay design and the inlay retained bridges with tub design exhibited $0 \%$ secondary caries.

Table 1: Comparison between the inlay-shaped and the tub-shaped groups after 1 year follow-up

\begin{tabular}{lccl}
\hline & $\begin{array}{l}\text { Inlay-shaped } \\
\text { group (\%) }\end{array}$ & $\begin{array}{l}\text { Tub-shaped } \\
\text { group (\%) }\end{array}$ & p value \\
\hline Retention & 93.3 & 80 & 0.598 \\
$\begin{array}{l}\text { Marginal adapta- } \\
\text { tion }\end{array}$ & 100.0 & 100.0 & - \\
Secondary caries & 0.0 & 0.0 & - \\
\hline
\end{tabular}

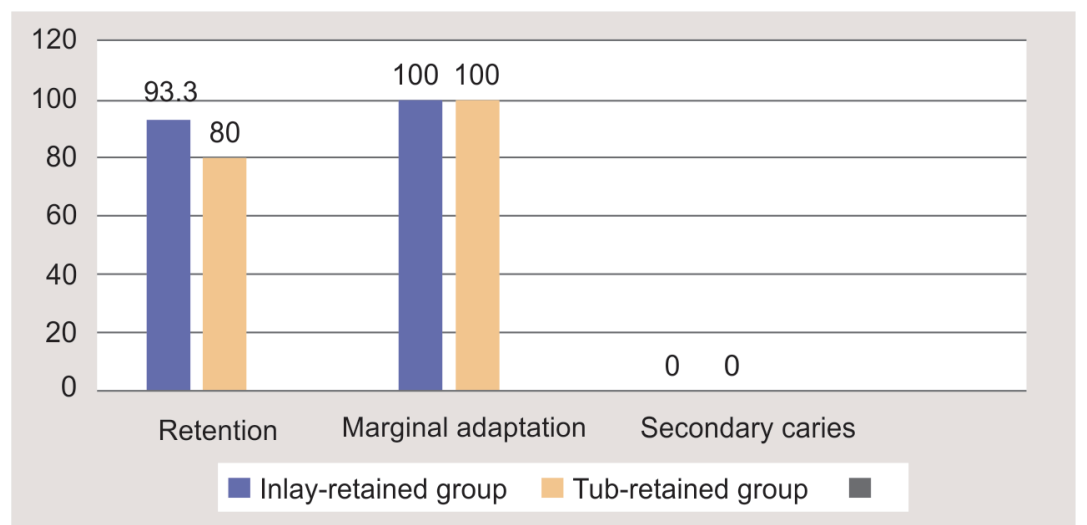

Fig. 4: Bar chart representing the comparison between the inlay-shaped and the tub-shaped groups after 1-year follow-up 


\section{Discussion}

The advantages of inlay bridges over conventional ones include conserving the tooth structure, preserving the pulp health, more accessible margins for finishing, and less susceptibility for gingival irritation. $^{7}$

Conventional bridges showed superior strength properties when compared to inlay bridges, with the later showed fracture failures of about $50 \%$ of the total failures, while the former showed $0 \%$. The weakest points of the inlay bridges were the interface between the tooth and the restoration and at the isthmus portion of the inlay. The final surface finish, restoration design, fabrication technique, composition, and microstructure of ceramic materials are among the several factors that control the strength of the restorations. ${ }^{8}$

Two designs of the inlay retained bridges were assessed: the inlay- and the tub-shaped preparation designs. The preparations were done according to the guidelines mentioned in the literature, by the same operator for standardization. ${ }^{9}$

The final impression was done using addition silicon (polyvinyl siloxane) as it offers excellent dimensional stability, high tear strength, good working and setting times, excellent wettability, automixed system, extremely high accuracy even superior to that of the digital impressions, minimal distortion on removal, and dimensionally stable even after 1 week. ${ }^{10}$

The two-step impression technique was selected as it is more accurate, with smaller discrepancies and longer term dimensional stability than the one-step impression technique. ${ }^{11}$

The extraoral scanner was used for scanning the master cast for designing the restoration using the computer. These scanners provide reliable, accurate, and fast scanning, with no intraoral restrictions (such as blood, saliva, and tongue movement). It used blue light technology, which enables quick scanning and provides high precision. ${ }^{12}$

Polishing of zirconia was done after occlusal adjustments rather than glazing. Glazing decreases the flexural strength of monolithic zirconia. Polishing produced a smooth surface that causes less wear of the opposing natural enamel when compared to glazing. ${ }^{13}$

After intraoral checking of inlay retained zirconia restorations, noncovalent adsorption of salivary proteins occurs. Only spraying the fitting surfaces of the restorations using water will lead to a significant reduction in the strength of the bond between the zirconia and the tooth structure. Sandblasting was effective in the removal of salivary proteins with no improvement in the bond strength. Zirconia surface cleaning material was used to effectively remove the saliva remnants and to improve the bond between the resin cement and the zirconia. ${ }^{14}$

Air abrasion with $50 \mu \mathrm{m} \mathrm{Al} \mathrm{O}_{3}$ for 15 seconds at 2.5 bars was able to create irregularities on the surface of zirconia, when combined with cements containing phosphate ester monomer, leading to improvement in the bond strength, as the phosphate ester monomer forms a chemical bond with metallic oxides such as zirconia oxides. ${ }^{15}$

Self-adhesive resin cement was utilized owing to its advantages, including reduction in postoperative sensitivity, more time-saving by eliminating the etching step, the light curing can be done via the bridge material and avoidance of air bubble inclusion during cementation by using automixing tips and double-barrel tubes. ${ }^{16}$

\section{Retention}

Retention form is considered a key factor for restoration success. There are many factors starting from size and geometry of the prepared teeth, irregularities of the intaglio surface of the restoration, type of cement used, and the film thickness of luting agent that can affect the retention in fixed prosthodontics. ${ }^{17}$

In the present study, it was shown that the overall retention rate in 1 year for the inlay-shaped design was $93.3 \%$ and for the tub-shaped design was $80 \%$, and this difference was statistically insignificant.

The results of this study were in agreement with Chaar et al. ${ }^{18}$ who conducted a study in 2015 , assessing the performance of posterior zirconia inlay retained bridges and found that the survival rate of inlay retained bridges was $95.8 \%$. Debonding was reported for two inlay retained bridges (6.9\%) after 1 year.

Finally, the results of this study were not in agreement with Sailer et al. ${ }^{19}$ who conducted a study in 2013 to evaluate the clinical success of resin-bonded fixed partial dentures. The author found that the retention rate of the bridges was $100 \%$ with no debonding of any case. These differences in the results may be related to the difference in the technique of bonding, materials used for bonding, the material of the bridges, and the design of the resin bonded bridge.

\section{Marginal Fit}

Excellent marginal adaptation extends the longevity of restorations. The presence of a gap in the marginal area may lead to cement solubility and plaque retention, producing harmful consequences for both the tooth and the surrounding supporting structures. It secures the cement from excessive exposure to the oral cavity fluids, leading to slow disintegration of the cement chemical, physical, and mechanical characteristics that may lead to microleakage, secondary caries, tooth discoloration, and cracking of the restoration. ${ }^{20}$

The preciseness of the marginal adaptation of restorations can be influenced by many variables including the impression material used, the impression technique, the accuracy of the scanner, the processing of the geometrical data collected, and the methodology of transforming the data to construct a digital model by the software. ${ }^{21}$

It was shown, in this trial, that the overall marginal adaptation in 1 year for the inlay-shaped design was $100 \%$ and for the tubshaped design was $100 \%$, and no difference was observed between both groups.

The results of this study were in agreement with Harder et al. ${ }^{22}$ who conducted a study in 2010 to clinically evaluate the outcome of inlay retained bridges after 8 years of service and found that marginal adaptation quality for the bridges were given successful and surviving scores without any recorded failures.

The results of this study were not in agreement with Sailer et al. ${ }^{19}$ who conducted a study in 2013 to clinically evaluate the survival rates and complication rates of resin bonded bridges and found that most of the reconstructions showed a fair marginal adaptation. Also, the author found that the clinically acceptable marginal gaps were found in $68.6 \%$ of the bridges.

\section{Secondary Caries}

Secondary caries at the restoration margins has been reported as the most common cause of failure. Early tooth decay may lead to pathological changes in the pulp, while deep caries may result in large damage to the hard dental structures. ${ }^{23}$

Secondary caries mainly occurs on the margin interfaces, this may be attributed to plaque accumulation and interference with its removal in these areas. ${ }^{24}$ 
In the present study, it was shown that the overall occurrence of secondary caries in 1 year for the inlay-shaped design was $0 \%$ and for the tub-shaped design was $0 \%$, and no difference was observed between both groups.

The results of this study were in agreement with Al-Moaleem et al. ${ }^{9}$ in 2014 who conducted a study for the evaluation of the outcome and the survival rates of inlay retained bridges, and they found that the secondary caries was not detected during the evaluation period.

The results of this study were not in agreement with Harder et al. ${ }^{22}$ in 2010 who conducted a study to assess bridges with inlay retainer constructed using pressable ceramics and found that failure happened due to secondary caries at the abutment teeth. This difference may be due to different materials of the bridges and different types of resin cement used.

Food impaction in relation to fixed prostheses is a common complaint that may result in gingival and periodontal diseases. With proper pontic-ridge relation and proper pontic contour, food accumulation can be avoided. Moreover, for maxillary restorations, the pontic design was modified ridge lab, while for the mandibular restorations the design was sanitary. ${ }^{25}$

To summarize, the aim of this trial was to clinically assess the tub design and the inlay design in cases with missed posterior tooth regarding retention, marginal adaptation, and secondary caries occurrence after 1 year of service. The results clarified that the inlay-shaped design shows superior retention compared to the tub design, and this may be attributed to the larger surface area involved for bonding in the inlay-shaped group than that involved in the tub-shaped group. Regarding marginal fit and secondary caries occurrence, both designs show comparable results. This may be due to making an accurate impression by adding silicon, scanning the master cast rather than the impression, utilizing a scanner with blue light technology, milling the zirconia at its pre-sintered stage, absence of any veneering materials, the short length of the span, and the property of fluoride and calcium release from the resin cement. However, no statistically significant difference was observed between other groups $24,26,27$

One limitation of this study is the follow-up period of 1 year. More information about the outcomes of the restorations can be gathered over large numbers of patients. Another limitation is the difficulty related to constructing both designs in the same patient, i.e., case selection with such requirements.

\section{CONCLUSION}

Within the limitations of this study, we can conclude that monolithic zirconia inlay retained bridges with inlay and tub designs cemented with resin cement represented a clinically successful restoration. Regarding retention, marginal adaptation, and secondary caries occurrence, both inlay designs exhibited comparable outcomes.

\section{Clinical Recommendation}

The use of the inlay retained bridges with tub design can be superior to the inlay retained bridges with inlay design, regarding the conservation of the tooth structure with the same retention rate, marginal adaptation, and occurrence of secondary caries.

\section{Ethics Approval and Consent to Participate}

This trial was an in vivo, randomized control trial approved by the Ethics Committee, Faculty of Dentistry-Cairo University. All participants have signed the consent in order to participate in this trial.

\section{Consent for Publication}

I declare that the article is original and has not been published in any other journal.

\section{ACKnOWLedgments}

I would like to thank Professor Omaima Salah El Dein El Mahallawi and Dr Ahmed Nabil for their support.

\section{References}

1. Aljehani YA, Baskaradoss JK, Geevarghese A, et al. Current trends in aesthetic. Dentist Health (Irvine Calif) 2014;6(August):1941-1949.

2. Madfa AA, Al-sanabani FA, Al-qudami $\mathrm{NH}$, et al. Use of zirconia in dentistry: an overview. Biomater J 2014;5:1-9.

3. Liu Y, Feng H, Bao Y, et al. Fracture and interfacial delamination origins of bilayer ceramic composites for dental restorations. J Eur Ceram Soc 2010;30(6):1297-1305. DOI: 10.1016/j.jeurceramsoc.2009.11.019.

4. Mehta $D$, Shetty R. Bonding to zirconia: elucidating the confusion. Int Dent 2010;12(2):1-7.

5. Ohlmann B, Rammelsberg P, Schmitter M, et al. All-ceramic inlayretained Fixed partial dentures: preliminary results from a clinical study. J Dent 2008;36(9):692-696. DOI: 10.1016/j.jdent.2008.04.017.

6. Deniz A, Kale E. A prospective cohort study on cast-metal slotretained resin-bonded fixed dental prostheses in single missing first molar cases: results after up to 7.5 years. J Adhes Dent 2013;15(1): 73-84.

7. Abdulkarim A, Samran A, Aswad M, et al. A new design for posterior inlay-retained fixed partial denture. J Prosthodont Res 2013;57(2):146149. DOI: 10.1016/j.jpor.2012.09.005.

8. Wolfart S, Bohlsen F, Wegner SM, et al. A preliminary prospective evaluation of all-ceramic crown-retained and inlay-retained fixed partial dentures. Int J Prosthodont 2005;18(6):497-505.

9. Al-Moaleem MM. Clinical evaluation of porcelain fused to metal inlay-retained bridges. J Dent Med Sci 2014;13(9):129-133. DOI: 10.9790/0853-1396129133.

10. Takeuchi $Y$, Koizumi H, Furuchi $M$, et al. Use of digital impression systems with intraoral scanners for fabricating restorations and fixed dental prostheses. J Oral Sci 2018;60(1):1-7. DOI: 10.2334/josnusd.170444.

11. Jamshidy L, Mozaffari HR, Faraji P, et al. Accuracy of the one-stage and two-stage impression techniques: a comparative analysis. Int J Dent 2016;2016.

12. Rudolph $\mathrm{H}$, Salmen $\mathrm{H}$, Moldan $\mathrm{M}$, et al. Accuracy of intraoral and extraoral digital data acquisition for dental restorations. J Appl Oral Sci 2016;24(1):85-94. DOI: 10.1590/1678-775720150266.

13. Kumchai $H$, Juntavee $P$, Sun $A F$, et al. Effect of glazing on flexural strength of full-contour zirconia. Int J Dent 2018;2018 $10.1155 / 2018 / 8793481$.

14. Kim D-H, Son J-S, Jeong S-H, et al. Efficacy of various cleaning solutions on saliva-contaminated zirconia for improved resin bonding. J Adv Prosthodont 2015;7(2):85. DOI: 10.4047/jap.2015.7.2.85.

15. Dalla $R$, Mattiello $L$, Marcos $T$, et al. A review of surface treatment methods to improve the adhesive cementation of zirconia-based ceramics. 2013;2013.

16. Saad DED, Atta O, El-Mowafy O. The postoperative sensitivity of fixed partial dentures cemented with self-adhesive resin cements a clinical study. J Am Dent Assoc 2010;141(12):1459-1466. DOI: 10.14219/jada. archive.2010.0108.

17. Narula S, Punia V, Khandelwal $M$, et al. Retention in conventional fixed partial dentures: a review. J Clin Diagnostic Res 2011;5(5):1128-1133.

18. Chaar MS, Kern M. Five-year clinical outcome of posterior zirconia ceramic inlay-retained FDPs with a modified design. J Dent 2015;43(12):1411-1415. DOI: 10.1016/j.jdent.2015.11.001. 
19. Sailer I, Bonani T, Brodbeck U, et al. Retrospective clinical study of single-retainer cantilever anterior and posterior glass-ceramic resin-bonded Fixed dental prostheses at a mean follow-up of 6 years. Int J Prosthodont 2013;26(5):443-450. DOI: 10.11607/ ijp.3368.

20. Tjan AH, Sarkissian R. Effect of preparation finish on retention and fit of complete crowns. J Prosthet Dent 1986;56(3):283-288. DOI: 10.1016/0022-3913(86)90004-1.

21. Örtorp A, Jönsson D, Mouhsen A, et al. The fit of cobalt-chromium three-unit Fixed dental prostheses fabricated with four different techniques: a comparative in vitro study. Dent Mater 2011;27(4): 356-363. DOI: 10.1016/j.dental.2010.11.015.

22. Harder S, Wolfart S, Eschbach S, et al. Eight-year outcome of posterior inlay-retained all-ceramic fixed dental prostheses. J Dent 2010;38(11):875-881. DOI: 10.1016/j.jdent.2010.07.012.
23. Sundh B, Odman P. A study of fixed prosthodontics performed at a university clinic 18 years after insertion. J Prosthodont 1997;10(6): 513-519.

24. Arzu Zeynep YB, Unver S. Etiology of secondary caries in prosthodontic treatments. Intech Open 2018;1(8):13.

25. Nagarsekar A, Gaunkar R, Aras M. Knowledge, attitude, and practice of dental professionals regarding the effect and management of food impaction associated with fixed partial denture prostheses: a survey. J Indian Prosthodont Soc 2016;16(4):372-379. DOI: 10.4103/0972 4052.191286

26. Medina-Sotomayor P, Pascual-Moscardó A, Camps I. Accuracy of four digital scanners according to scanning strategy in complete-arch impressions. PLoS ONE 2018;13(9):e0202916. DOI: 10.1371/journal. pone.0202916.

27. Lally U. Resin-bonded fixed partial dentures past and present--an overview. J Ir Dent Assoc 2013;58(6):294-300. 